\title{
Molecular-genetic mechanisms in development of degree of function and hyperplasia of thyroid gland of patients with nodular goiter with autoimmune thyroiditis and thyroid adenoma
}

\begin{abstract}
Michael I. SHEREMET ${ }^{1}$, Larisa P. SYDORCHUK ${ }^{3}$, Viktor O. SHIDLOVSKYI ${ }^{2}$, Olexandr V. SHIDLOVSKYI ${ }^{2}$, Natalia A. SHEREMET ${ }^{4}$, Nina P. TKACHUK ${ }^{1}$, Vitaliy V. MAKSYMYUK ${ }^{2}$, Volodimir V. TARABANCHUK ${ }^{1}$, Yan V. GYRLA ${ }^{1}$, Oleksandr V. BILOOKYI ${ }^{1}$, Viktor P. DOROSH ${ }^{5}$

${ }^{1}$ Surgery Department No1, Bukovinian State Medical University, Ukraine

${ }^{2}$ Surgery Department, I.Y. Horbachevsky State Medical University, Ukraine ${ }^{3}$ Department of Family Medicine, Bukovinian State Medical University, Ukraine ${ }^{4}$ First City Polyclinic, Chernivtsi, Ukraine ${ }^{5}$ Surgery Department, The Regional Clinical Hospital, Chernivtsi, Ukraine
\end{abstract}

\section{ABSTRACT}

Introduction. Autoimmune thyroiditis affects in average $2 \%$ to $5 \%$ of the general population, with young adult females and the elderly being the most vul-nerable patients. Hashimoto's thyroiditis causing hypothyroidism is the most prevalent etiology. Although genetics is well known to cause and influence the progression of autoimmune diseases in approximately 79\%, other environmental factors are known to be involved in the development of autoimmune thyroid dis-eases: quantity of ingested iodine, stress, drugs, pregnancy, and changes in sexual hormones.

Autoimmune thyroiditis, as a background disease of nodular goiter, in which hypothyroidism usually develops, has been insufficiently studied.

Material and methods. The BCL-2 (rs17759659), CTLA-4 (rs231775), Fas (rs2234767) genes' polymorphism were studied by Real-Time Polymerase Chain Reaction in 95 patients with NGAIT, 30 patients with thyroid adenoma (TA) and 25 healthy individuals. The thyroid gland (TG) functional activity changes (nor-mal function, subclinical and clinical hypothyroidism) and TG hyperplasia degrees (IB, II and III) were analyzed.

Results. TA and NGAIT are more common in the minor G-allele carriers (GA-and GG-genotypes) of the $B C L-2$ gene and in homozygous G allele patients (GG-genotype) of the Fas gene by 11.5 and 4.34 times $(p<0.01)$, with no signifi-cant interdependence between the CTLA4 gene's genotypes. TG hyperplasia in patients' general cohort as well as in those with NGAIT is associated with the wild A alleles of the CTLA-4 gene (AA- and AG-genotypes): the I and III degree hy-perplasia occurred reliably more frequently in the AA genotype carriers, and II degree of the TG enlargement in the AG genotype patients.

Conclusions. Pathology of the thyroid gland has unreliable chances to be inherited depending on the polymorphism of BCL-2 (rs17759659), CTLA-4 (rs231775) and Fas (rs2234767) genes in Bukovina region (Western Ukraine). We did not find any difference between the relative incidences of the genotypes of the analyzed genes in the patients with NGAIT and those with TA or depending on the TG function (euthyroid goiter, subclinical and clinical hypothyroidism)..
\end{abstract}

Keywords: nodular goiter with autoimmune thyroiditis, thyroid adenoma, functional state, hyperplasia, polymorphisms of APO-1 / FAS, CTLA-4 and BCL-2 genes 


\author{
Abbreviations \\ NGAIT - nodular goiter with autoimmune thyroiditis \\ AIT - autoimmune thyroiditis \\ TG - thyroid gland, TA - thyroid adenoma
}

\section{INTRODUCTION}

Autoimmune thyroiditis and nodular goiter represent very dynamic yet recent subjects in the field of interdisciplinary approach involving endocrinologists, oncologists, surgeons, geneticists, as well was pathologists (1-8). The clinical evaluation starts from the discovery of a thyroid nodule $(4,5)$.

Nodular goiter is considered as a non-autoimmune thyroid disease, and there have been findings to support this hypothesis (4-6). However, several immunological alterations have been found in these patients, such as HLA-DR antigen expression in thyrocytes, the presence of growth-stimulating immunoglobulins, and an increase in dendritic cells and lymphocytes, which suggest the possibility of autoimmune problems $(1,8-10)$. Although many of these findings may be an epiphenomenon of other primary defects in immunoregulation, the alteration of lymphocyte populations indicates a primary defect $(6,7,10,12$ 18). Variations in several genes have been studied as possible risk factors for Hashimoto thyroiditis. Some of these genes are part of a family called the human leukocyte antigen (HLA) complex (8-10). The HLA complex helps the immune system distinguish the body's own proteins from proteins made by foreign invaders (such as viruses and bacteria). Other genes that have been associated with AIT help regulate the immune system or are involved in normal thyroid function. Most of the genetic variations that have been discovered are thought to have a small impact on a person's overall risk of developing this condition (14-18).

AIT thought to result from a combination of genetic and environmental factors (1-3, 8-18). Some of these factors have been identified, but many remain unknown (19-24).

AIT is an autoimmune disorder, one of a large group of conditions that occur when the immune system attacks the body's own tissues and organs (23). This shortage of thyroid hormones underlies the signs and symptoms of Hashimoto thyroiditis. However, some individuals with thyroid antibodies never develop hypothyroidism or experience any related signs or symptoms (24).

Other, nongenetic factors also play a role in Hashimoto thyroiditis. These factors may trigger the condition in people who are at risk, although the mechanism is unclear. Potential triggers include changes in sex hormones (particularly in women), viral infections, certain medications, exposure to ionizing radiation, and excess consumption of iodine, a substance involved in thyroid hormone production $(2,24)$.

In Ukraine, the polymorphism of genes associated with apoptosis of thyrocytes, lymphoid cells, or regulation of T cell activation (Bcl-2, Fas (APO-1), CTLA-4) in NGAIT and TA had not been studied previously, while there may be several national and local peculiarities.

\section{MATERIAL AND METHODS}

Ninety-five women with NGAIT underwent examination during 2013 to 2016, based on Chernivtsi Regional Hospital, Ukraine. The age of patients ranged from 23 to 72 years. Diagnosis is exposed based on clinical and laboratory examinations (thyroid peroxidase antibodies (ATPO) - 60$250 \mathrm{U} / \mathrm{ml}$ thyroglobulin antibody (ATTH) $-60-500$ $\mathrm{U} / \mathrm{ml}$; thyroid-stimulating hormone (TSH) -4.10 $\mathrm{IU} / \mathrm{I})$, and thyroid sonography and histologic confirmation after surgical operation.

The group of 30 women passed selection based on ultrasound, fine needle aspiration puncture biopsy and thyroid histological conclusion after surgery diagnosed with thyroid adenoma. We have identified this group because this pathology is one of the most common forms of nodular goiter. Control group included 25 healthy donors.

Genetic studies performed in the laboratory of genetics at the "Nicolae Testemițanu" State University of Medicine and Pharmacy Chisinau (Republic of Moldova). DNA was extracted from whole venous blood lymphocytes. Venous blood was stored in test tubes, stabilized with K2-EDTA. Isolation and purification of DNA from the material obtained was performed according to methodological guidance of Thermo Scientific GeneJET Genomic DNA Purification kit (\#K0721, Thermo Fisher Scientific).

\section{Quantitative Real-Time PCR (RT-PCR)}

Polymerase chain reaction (PCR) was performed in real-time (RT-PCR) using Taq-DNA polymerase and specific primers on QuantStudio 6 equipment, Applied Biosystems (USA), which allowed us to obtain amplicons to determine their number in "real time" and reduce the likelihood of diagnostic error. Analysis of the data was performed using the Quant Studio Real Time Software (Fig. 1-3). 


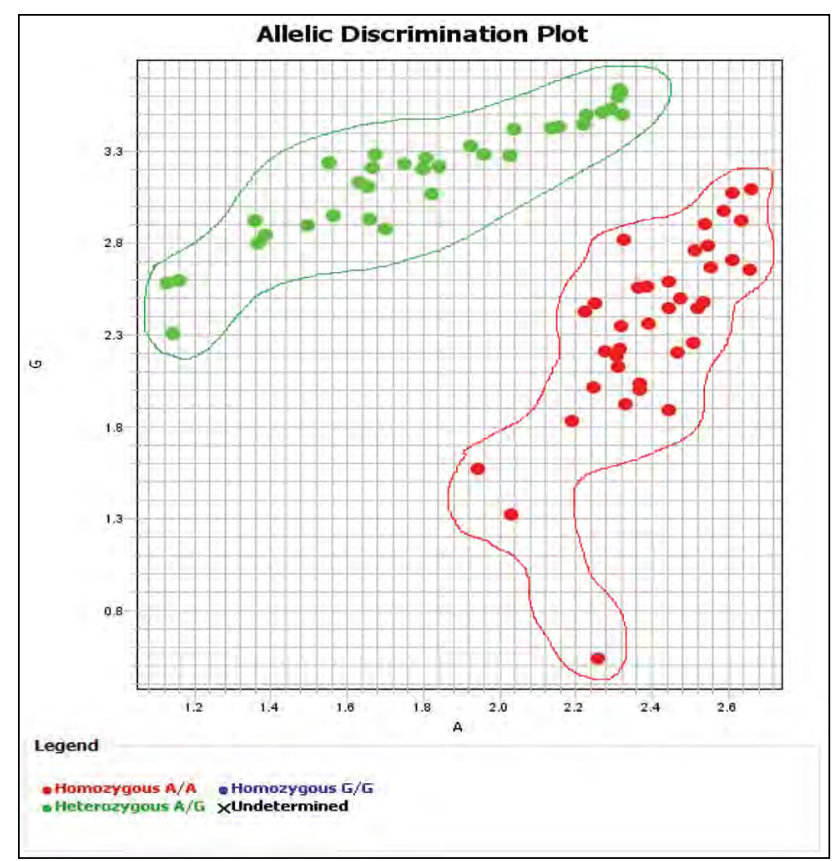

FIGURE 1. CTLA4 gene polymorphism (rs 231775) alleles discrimination

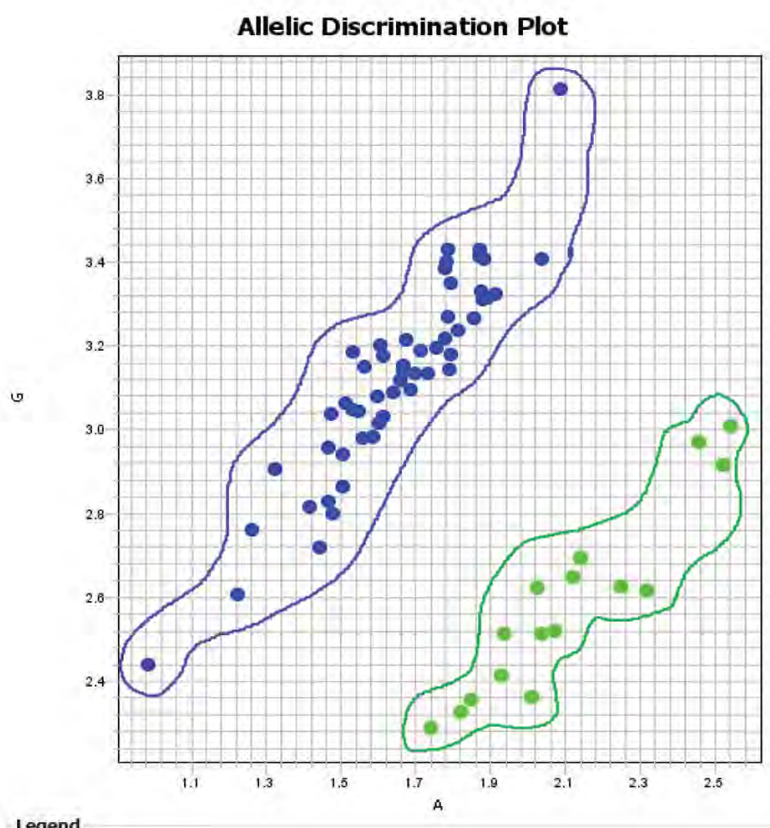

- Homozygous A/A e Homozygous G/E - Heterozygous $A / G$ XUndetermined

FIGURE 3. FAS gene polymorphism (rs 2234767) alleles discrimination

\section{STATISTICAL ANALYSIS}

Statistical analysis was performed using Statistica 7.0 (Stat Soft Inc., USA) software. Nominal data presented in the form of quantitative and

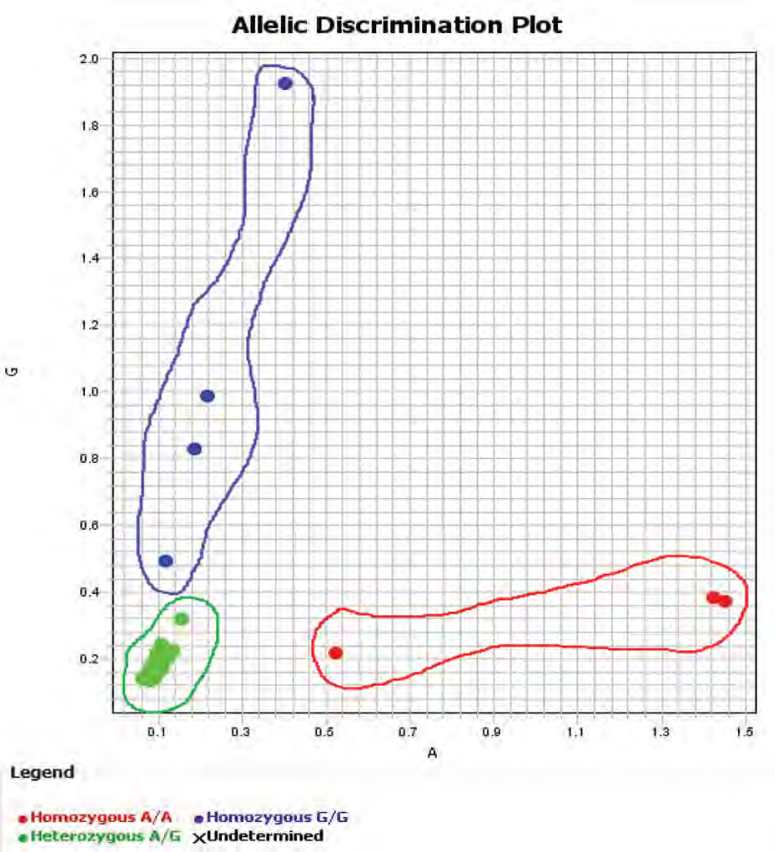

FIGURE 2. BCL-2 gene polymorphism (17759659) alleles discrimination

percentages. For the genotypes distribution comparison used Pearson's criterion $\left(c^{2}\right)$. Analysis of qualitative data (categorical variables), risk of thyroid pathology development was assessed using a binary logistic regression model using the relative risk (ReIR), risk ratio (RR) and odds ratio (OR) with 95\% confidence interval [95\% $\mathrm{Cl}$ ], chi-square test $\left(\chi^{2}\right)(d f=1)$. The difference was considered reliable at $p<0.05$.

\section{RESULTS AND DISCUSSIONS}

Analysis of the incidence of conditions, considering the thyroid function, (euthyroid goiter, subclinical and clinical hypothyroidism) did not reveal a reliable difference depending on the genotype of the CTLA-4 ( $r$ 231775), BCL-2 (rs17759659) and APO-1 / Fas (rs2234767) genes (tab. 1). At the same time, we established the dominance of BCL2 gene's GA-genotype over AA- and GG-genotypes by 6.5 and 25.97 times $(p<0.001)$ regardless of the thyroid function. A-allele of the CTLA-4 gene dominated over the G-allele 2.65 and 2.30 times $(p<0.001)$ in patients with NGAIT and TA with euthyroid goiter and subclinical hypothyroidism. GG-genotype of the CTLA-4 gene was observed in patients with clinical hypothyroidism. In all patients regardless of the thyroid function, GG-genotype of the Fas gene predominant the AG-genotype by 3.18-10.5 times ( $<<0.001)$, respectively. 
TABLE 1. The distribution of the polymorphic variants of the BCL-2 (rs17759659), CTLA-4 (rs231775) and APO-1/Fas (rs2234767) genes in patients with thyroid pathology considering its function

\begin{tabular}{|c|c|c|c|c|c|c|}
\hline \multirow{2}{*}{\multicolumn{2}{|c|}{ The genes under study, n (\%) }} & \multirow{3}{*}{$\begin{array}{c}\text { Control, } n=25(\%) \\
3(12.0)\end{array}$} & \multicolumn{3}{|c|}{ The thyroid gland function } & \multirow{3}{*}{$\begin{array}{c}c^{2} \\
p \\
c^{2}=1.57 p>0.05\end{array}$} \\
\hline & & & \multirow{2}{*}{$\begin{array}{c}\begin{array}{c}\text { Euthyroid goiter, } \\
n=31\end{array} \\
4(12.90) \\
\end{array}$} & \multirow{2}{*}{$\begin{array}{c}\begin{array}{c}\text { Subclinical hypo- } \\
\text { thyroidism, n=71 }\end{array} \\
4(5.63)\end{array}$} & \multirow{2}{*}{$\begin{array}{c}\text { Clinical hypothy- } \\
\text { roidism, } n=23\end{array}$} & \\
\hline \multirow{3}{*}{$B C L-2(\mathrm{~A} / \mathrm{G}), \mathrm{n}(\%)$} & $A A$ & & & & & \\
\hline & $G A$ & $21(84.0)$ & $26(83.87)$ & $65(91.55)$ & $19(82.61)$ & $c^{2}=1.98 p>0.05$ \\
\hline & $G G$ & $1(4.0)$ & $1(3.23)$ & $2(2.82)$ & $2(8.70)$ & $c^{2}=1.63 p>0.05$ \\
\hline \multicolumn{2}{|l|}{$\begin{array}{l}c^{2} \\
p\end{array}$} & $\begin{array}{l}c^{2}=64,80 \\
p<0,001\end{array}$ & $c^{2}=54.10 p<0.001$ & $c^{2}=162.55 p<0.001$ & $c^{2}=37.70 p<0.001$ & - \\
\hline \multirow{3}{*}{$\begin{array}{c}\text { CTLA-4 } \\
(+49 \mathrm{G} / \mathrm{A}), \mathrm{n}(\%)\end{array}$} & $A A$ & $15(60.0)$ & $15(48.39)$ & $31(43.66)$ & $13(56.52)$ & $c^{2}=1.18 p>0.05$ \\
\hline & $A G$ & $9(36.0)$ & $15(48.39)$ & $37(52.11)$ & $10(43.48)$ & $c^{2}<1.0 p>0.05$ \\
\hline & GG & $1(4.0)$ & $1(3.23)$ & $3(4.23)$ & 0 & $p>0.05$ \\
\hline \multicolumn{2}{|l|}{$\begin{array}{l}c^{2} \\
p\end{array}$} & $\begin{array}{l}c^{2}=36,48 \\
p<0,001\end{array}$ & $c^{2}=18.67 p<0.001$ & $\begin{array}{l}c^{2}=41.75 \\
p<0.001\end{array}$ & $c^{2}<1.0 p>0.05$ & - \\
\hline \multirow{2}{*}{$\begin{array}{c}\text { Fas } \\
(-1377 \mathrm{G} / \mathrm{A}), \mathrm{n}(\%)\end{array}$} & GA & $6(24.0)$ & $4(12.90)$ & $17(23.94)$ & $2(8.70)$ & \multirow{2}{*}{$c^{2}=3.52 p>0.05$} \\
\hline & GG & $19(76.0)$ & $27(87.10)$ & $54(76.06)$ & $21(91.30)$ & \\
\hline \multicolumn{2}{|l|}{$\begin{array}{l}c^{2} \\
p\end{array}$} & $\begin{array}{l}c^{2}=72,20 \\
p<0,001\end{array}$ & $c^{2}=34.13 p<0.001$ & $\begin{array}{l}c^{2}=38.56 \\
p<0.001\end{array}$ & $c^{2}=31.39 p<0.001$ & - \\
\hline
\end{tabular}

TABLE 2. The distribution of polymorphic variants of the BCL-2 (rs17759659), CTLA-4 (rs231775) and APO-1 / Fas (rs2234767) genes in patients with thyroid adenoma, considering its features

\begin{tabular}{|c|c|c|c|c|c|c|}
\hline \multirow{2}{*}{\multicolumn{2}{|c|}{ The genes under study, n (\%) }} & \multirow{3}{*}{$\begin{array}{c}\text { Control, } \mathrm{n}=25(\%) \\
3(12.0)\end{array}$} & \multicolumn{3}{|c|}{ The thyroid gland function, $\mathrm{n}(\%)$} & \multirow{3}{*}{$\begin{array}{l}c^{2} \\
p \\
-\end{array}$} \\
\hline & & & \multirow{2}{*}{$\begin{array}{c}\begin{array}{c}\text { Euthyroid goiter } \\
\mathrm{n}=10\end{array} \\
0 \\
\end{array}$} & \multirow{2}{*}{$\begin{array}{c}\begin{array}{l}\text { Subclinical hypo- } \\
\text { thyroidism, } \mathrm{n}=13\end{array} \\
1(7.69)\end{array}$} & \multirow{2}{*}{$\begin{array}{c}\begin{array}{c}\text { Clinical hypothy- } \\
\text { roidism, } n=7\end{array} \\
0 \\
\end{array}$} & \\
\hline \multirow{3}{*}{$B C L-2(\mathrm{~A} / \mathrm{G}), \mathrm{n}(\%)$} & $A A$ & & & & & \\
\hline & GA & $21(84.0)$ & $10(100.0)$ & $12(92.31)$ & $7(100.0)$ & $p>0.05$ \\
\hline & $G G$ & $1(4.0)$ & 0 & 0 & 0 & 0 \\
\hline \multicolumn{2}{|l|}{$\begin{array}{l}c^{2} \\
p\end{array}$} & $\begin{array}{l}c^{2}=64,80 \\
p<0,001\end{array}$ & - & $c^{2}=18.62 p<0.001$ & - & - \\
\hline \multirow{3}{*}{$\begin{array}{c}\text { CTLA-4 } \\
(+49 \mathrm{G} / \mathrm{A}), \mathrm{n}(\%)\end{array}$} & $A A$ & $15(60.0)$ & $5(50.0)$ & $3(23.08)$ & $4(57.14)$ & \multirow{2}{*}{$c^{2}=2.82 p>0.05$} \\
\hline & $A G$ & $9(36.0)$ & $5(50.0)$ & $10(76.92)$ & $3(42.86)$ & \\
\hline & GG & $1(4.0)$ & 0 & 0 & 0 & - \\
\hline \multicolumn{2}{|l|}{$\begin{array}{l}c^{2} \\
p\end{array}$} & $\begin{array}{l}c^{2}=36,48 \\
p<0,001\end{array}$ & - & $\begin{array}{l}c^{2}=7.54 \\
p=0.006\end{array}$ & $c^{2}<1.0 p>0.05$ & - \\
\hline \multirow{2}{*}{$\begin{array}{c}\text { Fas } \\
(-1377 \mathrm{G} / \mathrm{A}), \mathrm{n}(\%)\end{array}$} & $G A$ & $6(24.0)$ & 0 & $4(30.77)$ & $1(14.29)$ & \multirow{2}{*}{$c^{2}=3.89 p>0.05$} \\
\hline & GG & $19(76.0)$ & $10(100.0)$ & $9(69.23)$ & $6(85.71)$ & \\
\hline \multicolumn{2}{|l|}{$\begin{array}{l}c^{2} \\
p\end{array}$} & $\begin{array}{l}c^{2}=72,20 \\
p<0,001\end{array}$ & - & $\begin{array}{l}c^{2}=3.85 \\
p=0.049\end{array}$ & $p=0.015$ & - \\
\hline
\end{tabular}

In the patients with TA (table 2) and NGAIT (table 3), the distribution, taking into account the thyroid function, showed no statistically significant difference in the incidence of polymorphic variants of the genes analyzed.

I and III degree thyroid hyperplasia occurred much more frequently in individuals with AA-genotype CTLA-4 gene by $30.13 \%$ and $26.35 \%\left(c^{2}=9.26\right.$; $p=0.01$ ), whereas the II degree of the thyroid gland enlargement was more frequently recorded in the patients with $A G$-genotype by $33.52 \%$ and $34.04 \%$ $\left(c^{2}=12.34 ; p=0.002\right)$ respectively (tab. 4).

We have not found the reliable dependence of the thyroid hyperplasia degree on the polymorphic variants of the analyzed genes in the patients with TA (table 5).

In NGAIT patients, I and III degree hyperplasia was more often found in those having AA-geno- 
type of CTLA-4 gene than AG- and GG-genotype by $28.21 \%$ and $56.41 \% \quad\left(c^{2}=27.92 ; p<0.001\right)$ and $15.39 \%$ and $46.16 \%\left(c^{2}=12.92 ; p<0.001\right)$, respectively, as well as the II degree of the thyroid gland enlargement - by $31,54 \%$ and $23,85 \%\left(c^{2}=7,02\right.$; $p=0,03$ ) (tab. 6). On the other hand, the II degree hyperplasia was more frequently diagnosed in the individuals with $A G$-genotype, than in those with $A A-$ by $40.0 \%$ ( $\left.c^{2}=9.60 ; p=0.002\right)$, as well as more frequently than I and III degree thyroid hyperplasia by $36.67 \%$ and $31.54 \%\left(c^{2}=10.06 ; p=0.007\right)$.

In patients with euthyroid goiter I degree of thyroid hyperplasia was more common: in those with wild A-allele of $\mathrm{BCL}-2$ gene by $11,49-52,45 \%$ $(r \leq 0,029-0,001)$, A-allele of CTLA-4 gene - by 25.86 $35.35 \%\left(c^{2}=10.14-11.58 ; p=0.003-0.006\right)$, and favorable GG-genotype of the Fas gene by $46.43 \%$ and $60.03 \%\left(c^{2}=25.39 ; p<0.001\right)$ (tab. 7).

Patients with subclinical hypothyroidism were more often diagnosed with the II degree hyperplasia of the thyroid gland: in the carriers of intermediate genotype (AG) of BCL-2 gene and CTLA-4 gene, by $25.53 \%$ and $37.78 \%(r \leq 0.001)$ (tab. $7-8)$ and in the owners of favorable GG-genotype of Fas gene by $25.53 \%$ and $26.51 \%\left(c^{2}=11.33 ; p=0.003\right)$ (tab. 8$)$.

TABLE 3. The distribution of polymorphic variants of the BCL-2 (rs17759659), CTLA-4 (rs231775) and APO-1 / Fas (rs2234767) genes in patients with nodular forms of goiter against the background of autoimmune thyroiditis, considering the thyroid function

\begin{tabular}{|c|c|c|c|c|c|c|}
\hline \multirow{2}{*}{\multicolumn{2}{|c|}{ The genes under study, n (\%) }} & \multirow{3}{*}{$\begin{array}{c}\text { Control, } \mathbf{n = 2 5}(\%) \\
3(12.0)\end{array}$} & \multicolumn{3}{|c|}{ The thyroid gland function, $n(\%)$} & \multirow{3}{*}{$\begin{array}{c}\mathbf{c}^{2} \\
\mathbf{p} \\
c^{2}=3.67 \\
p>0.05\end{array}$} \\
\hline & & & \multirow{2}{*}{$\begin{array}{c}\begin{array}{c}\text { Euthyroid goiter, } \\
\mathbf{n}=\mathbf{2 1}\end{array} \\
4(19.05) \\
\end{array}$} & \multirow{2}{*}{$\begin{array}{c}\begin{array}{c}\text { Subclinical hypo- } \\
\text { thyroidism, } \mathbf{n}=58\end{array} \\
3(5.17)\end{array}$} & \multirow{2}{*}{$\begin{array}{c}\begin{array}{c}\text { Clinical hypothy- } \\
\text { roidism, } n=16\end{array} \\
2(12.50)\end{array}$} & \\
\hline \multirow{3}{*}{$B C L-2(A / G), n(\%)$} & $A A$ & & & & & \\
\hline & $G A$ & $21(84.0)$ & $16(76.19)$ & $53(91.38)$ & $12(75.0)$ & $\begin{array}{l}c^{2}=4.44 \\
p>0.05\end{array}$ \\
\hline & GG & $1(4.0)$ & $1(4.76)$ & $2(3.45)$ & $2(12.50)$ & $\begin{array}{l}c^{2}=2.11 \\
p>0.05\end{array}$ \\
\hline \multicolumn{2}{|l|}{$\begin{array}{l}c^{2} \\
p\end{array}$} & $\begin{array}{l}c^{2}=64.80 \\
p<0.001\end{array}$ & $\begin{array}{l}c^{2}=27.0 \\
p<0.001\end{array}$ & $\begin{array}{l}c^{2}=131.9 \\
p<0.001\end{array}$ & $\begin{array}{l}c^{2}=18.75 \\
p<0.001\end{array}$ & - \\
\hline \multirow{3}{*}{$\begin{array}{c}\text { CTLA-4 } \\
(+49 \mathrm{G} / \mathrm{A}), \mathrm{n}(\%)\end{array}$} & $A A$ & $15(60.0)$ & $\begin{array}{c}10 \\
(47.62) \\
\end{array}$ & $28(48.28)$ & $9(56.25)$ & $c^{2}<1.0 p>0.05$ \\
\hline & $A G$ & $9(36.0)$ & $10(47.62)$ & $27(46.55)$ & $7(43.75)$ & $c^{2}<1.0 p>0.05$ \\
\hline & GG & $1(4.0)$ & $1(4.76)$ & $3(5.17)$ & 0 & $c^{2}<1.0 p>0.05$ \\
\hline \multicolumn{2}{|l|}{$\begin{array}{l}c^{2} \\
p\end{array}$} & $\begin{array}{l}c^{2}=36.48 \\
p<0.001\end{array}$ & $\begin{array}{l}c^{2}=11.57 \\
p=0.003\end{array}$ & $\begin{array}{l}c^{2}=31.09 \\
p<0.001\end{array}$ & $c^{2}<1.0 p>0.05$ & - \\
\hline \multirow{2}{*}{$\begin{array}{c}\text { Fas } \\
(-1377 \mathrm{G} / \mathrm{A}), \mathrm{n}(\%)\end{array}$} & $G A$ & $6(24.0)$ & $4(19.05)$ & $13(22.41)$ & $1(6.25)$ & \multirow{2}{*}{$\begin{array}{l}c^{2}=2.13 \\
p>0.05\end{array}$} \\
\hline & GG & $19(76.0)$ & $17(80.95)$ & $45(77.59)$ & $15(93.75)$ & \\
\hline \multicolumn{2}{|l|}{$\begin{array}{l}c^{2} \\
p\end{array}$} & $\begin{array}{l}c^{2}=72.20 \\
p<0.001\end{array}$ & $\begin{array}{l}c^{2}=16.10 \\
p<0.001\end{array}$ & $\begin{array}{l}c^{2}=35.31 \\
p<0.001\end{array}$ & $\begin{array}{l}c^{2}=24.50 \\
p<0.001\end{array}$ & - \\
\hline
\end{tabular}

TABLE 4. The distribution of polymorphic variants of the BCL-2 (rs17759659), CTLA-4 (rs231775) and APO-1 / Fas (rs2234767) genes in patients with thyroid disorders, taking into account the degree of its enlargement

\begin{tabular}{|c|c|c|c|c|c|c|}
\hline \multirow{2}{*}{\multicolumn{2}{|c|}{ The genes under study, n (\%) }} & \multirow{3}{*}{$\begin{array}{c}\text { Control, } \mathbf{n = 2 5}(\%) \\
3(12.0)\end{array}$} & \multicolumn{3}{|c|}{ The thyroid gland hyperplasia, $n$ (\%) } & \multirow{3}{*}{$\begin{array}{c}\mathbf{c}^{2} \\
\mathbf{p} \\
c^{2}=3.59 \\
p>0.05\end{array}$} \\
\hline & & & I degree, $n=59$ & II degree, $n=40$ & III degree, $n=26$ & \\
\hline \multirow{3}{*}{$B C L-2(\mathrm{~A} / \mathrm{G}), \mathrm{n}(\%)$} & $A A$ & & $5(8.47)$ & $1(2.50)$ & $4(15.38)$ & \\
\hline & GA & $21(84.0)$ & $52(88.14)$ & $38(95.0)$ & $20(76.92)$ & $\begin{array}{l}c^{2}=3.59 \\
p>0.05\end{array}$ \\
\hline & GG & $1(4.0)$ & $2(3.39)$ & $1(2.50)$ & $2(7.69)$ & $\begin{array}{l}c^{2}=1.21 \\
p>0.05\end{array}$ \\
\hline \multicolumn{2}{|l|}{$\begin{array}{l}c^{2} \\
p\end{array}$} & $\begin{array}{l}c^{2}=64.80 \\
p<0.001\end{array}$ & $\begin{array}{l}c^{2}=119.9 \\
p<0.001\end{array}$ & $\begin{array}{l}c^{2}=102.7 \\
p<0.001\end{array}$ & $\begin{array}{l}c^{2}=33.69 \\
p<0.001\end{array}$ & - \\
\hline \multirow{3}{*}{$\begin{array}{c}\text { CTLA-4 } \\
(+49 G / A), n(\%)\end{array}$} & $A A$ & $15(60.0)$ & $34(57.63)$ & $11(27.50)$ & $14(53.85)$ & $\begin{array}{l}c^{2}=9.26 \\
p=0.01\end{array}$ \\
\hline & $A G$ & $9(36.0)$ & $23(38.98)$ & $29(72.50)$ & $10(38.46)$ & $\begin{array}{l}c^{2}=12.34 \\
p=0.002\end{array}$ \\
\hline & GG & $1(4.0)$ & $2(3.39)$ & 0 & $2(7.69)$ & $p>0.05$ \\
\hline \multicolumn{2}{|l|}{$\begin{array}{l}c^{2} \\
p\end{array}$} & $\begin{array}{l}c^{2}=36.48 \\
p<0.001\end{array}$ & $\begin{array}{l}c^{2}=40.32 \\
p<0.001\end{array}$ & $\begin{array}{l}c^{2}=16.20 \\
p<0.001\end{array}$ & $\begin{array}{l}c^{2}=12.92 \\
p=0.002\end{array}$ & - \\
\hline \multirow{2}{*}{$\begin{array}{c}\text { Fas } \\
(-1377 \mathrm{G} / \mathrm{A}), \mathrm{n}(\%)\end{array}$} & $G A$ & $6(24.0)$ & $9(15.25)$ & $10(25.0)$ & $4(15.38)$ & \multirow{2}{*}{$\begin{array}{l}c^{2}=1,71 \\
p>0,05\end{array}$} \\
\hline & GG & $19(76.0)$ & $50(84.75)$ & $30(75.0)$ & $22(84.62)$ & \\
\hline \multicolumn{2}{|l|}{$\begin{array}{l}c^{2} \\
p\end{array}$} & $\begin{array}{l}c^{2}=72.20 \\
p<0.001\end{array}$ & $\begin{array}{l}c^{2}=56.98 \\
p<0.001\end{array}$ & $\begin{array}{l}c^{2}=20.0 \\
p<0.001\end{array}$ & $\begin{array}{l}c^{2}=24.92 \\
p<0.001\end{array}$ & - \\
\hline
\end{tabular}


TABLE 5. The distribution of polymorphic variants of the BCL-2 (rs17759659), CTLA-4 (rs231775) and APO-1 / Fas (rs2234767) genes in patients with adenoma of the thyroid gland, considering the degree of its enlargement

\begin{tabular}{|c|c|c|c|c|c|}
\hline \multirow{2}{*}{\multicolumn{2}{|c|}{ The genes under study, $\mathrm{n}(\%)$}} & \multirow{3}{*}{$\begin{array}{c}\text { Control, } \mathbf{n = 2 5}(\%) \\
3(12.0)\end{array}$} & \multicolumn{2}{|c|}{ The thyroid gland hyperplasia, $n$ (\%) } & \multirow{3}{*}{$\begin{array}{l}c^{2} \\
p \\
-\end{array}$} \\
\hline & & & \multirow{2}{*}{$\begin{array}{c}\text { I degree, } \mathbf{n}=\mathbf{2 0} \\
1(5.0)\end{array}$} & \multirow{2}{*}{ II degree, $n=10$} & \\
\hline \multirow{3}{*}{$B C L-2(A / G), n(\%)$} & $A A$ & & & & \\
\hline & $G A$ & $21(84.0)$ & $19(95.0)$ & $10(100.0)$ & $p>0.05$ \\
\hline & GG & $1(4.0)$ & 0 & 0 & 0 \\
\hline \multicolumn{2}{|l|}{$\begin{array}{l}c^{2} \\
p\end{array}$} & $\begin{array}{l}c^{2}=64.80 \\
p<0.001\end{array}$ & - & - & - \\
\hline \multirow{3}{*}{$\begin{array}{c}\text { CTLA-4 } \\
(+49 \mathrm{G} / \mathrm{A}), \mathrm{n}(\%)\end{array}$} & $A A$ & $15(60.0)$ & $10(50.0)$ & $2(20.0)$ & \multirow{2}{*}{$c^{2}<1,0 p>0,05$} \\
\hline & $A G$ & $9(36.0)$ & $10(50.0)$ & $8(80.0)$ & \\
\hline & GG & $1(4.0)$ & 0 & 0 & - \\
\hline \multicolumn{2}{|l|}{$\begin{array}{l}c^{2} \\
p\end{array}$} & $\begin{array}{l}c^{2}=36.48 \\
p<0.001\end{array}$ & - & $\begin{array}{l}c^{2}=7.20 \\
p=0.007\end{array}$ & - \\
\hline \multirow{2}{*}{$\begin{array}{c}\text { Fas } \\
(-1377 \mathrm{G} / \mathrm{A}), \mathrm{n}(\%)\end{array}$} & $G A$ & $6(24.0)$ & $4(20.0)$ & $1(10.0)$ & \multirow{2}{*}{$c^{2}<1,0 p>0,05$} \\
\hline & GG & $19(76.0)$ & $16(80.0)$ & $9(90.0)$ & \\
\hline \multicolumn{2}{|l|}{$\begin{array}{l}c^{2} \\
p\end{array}$} & $\begin{array}{l}c^{2}=72.20 \\
p<0.001\end{array}$ & $\begin{array}{l}c^{2}=14.40 \\
p<0.001\end{array}$ & $\begin{array}{l}c^{2}=12.80 \\
p<0.001\end{array}$ & - \\
\hline
\end{tabular}

TABLE 6. The distribution of polymorphic variants of the BCL-2 (rs17759659), CTLA-4 (rs231775) and APO-1 / Fas (rs2234767) genes in patients with nodular form of goiter against the background of autoimmune thyroiditis depending on the degree of the thyroid gland hyperplasia

\begin{tabular}{|c|c|c|c|c|c|c|}
\hline \multirow{2}{*}{\multicolumn{2}{|c|}{ The genes under study, $n(\%)$}} & \multirow{3}{*}{$\begin{array}{c}\text { Control, } \mathrm{n}=\mathbf{2 5}(\%) \\
3(12.0)\end{array}$} & \multicolumn{3}{|c|}{ The thyroid gland hyperplasia, $n$ (\%) } & \multirow{3}{*}{$\begin{array}{c}\mathbf{c}^{2} \\
\mathbf{p} \\
c^{2}=2.41 \\
p>0.05\end{array}$} \\
\hline & & & \multirow{2}{*}{$\begin{array}{c}\text { I degree , } n=39 \\
4(10.26) \\
\end{array}$} & \multirow{2}{*}{$\frac{\text { Il degree, } \mathbf{n = 3 0}}{1(3.33)}$} & \multirow{2}{*}{$\begin{array}{c}\text { III degree, } \mathbf{n}=\mathbf{2 6} \\
4(15.38)\end{array}$} & \\
\hline \multirow{3}{*}{$B C L-2(\mathrm{~A} / \mathrm{G}), \mathrm{n}(\%)$} & $A A$ & & & & & \\
\hline & $G A$ & $21(84.0)$ & $33(84.62)$ & $28(93.33)$ & $20(76.92)$ & $\begin{array}{l}c^{2}=3.01 \\
p>0.05\end{array}$ \\
\hline & GG & $1(4.0)$ & $2(5.13)$ & $1(3.33)$ & $2(7.69)$ & $c^{2}<1.0 p>0.05$ \\
\hline \multicolumn{2}{|l|}{$\begin{array}{l}c^{2} \\
p\end{array}$} & $\begin{array}{l}c^{2}=64,80 \\
p<0,001\end{array}$ & $\begin{array}{l}c^{2}=69.46 \\
p<0.001\end{array}$ & $\begin{array}{l}c^{2}=72.90 \\
p<0.001\end{array}$ & $\begin{array}{l}c^{2}=33.69 \\
p<0.001\end{array}$ & - \\
\hline \multirow{3}{*}{$\begin{array}{c}C T L A-4 \\
(+49 G / A), n(\%)\end{array}$} & $A A$ & $15(60.0)$ & $24(61.54)$ & $9(30.0)$ & $14(53.85)$ & $\begin{array}{l}c^{2}=7.02 \\
p=0.03\end{array}$ \\
\hline & $A G$ & 9 (36.0) & $13(33.33)$ & $21(70.0)$ & $10(38.46)$ & $\begin{array}{l}c^{2}=10.06 \\
p=0.007\end{array}$ \\
\hline & GG & $1(4.0)$ & $2(5.13)$ & 0 & $2(7.69)$ & $p>0.05$ \\
\hline \multicolumn{2}{|l|}{$\begin{array}{l}c^{2} \\
p\end{array}$} & $\begin{array}{l}c^{2}=36,48 \\
p<0,001\end{array}$ & $\begin{array}{l}c^{2}=27.92 \\
p<0.001\end{array}$ & $\begin{array}{l}c^{2}=9.60 \\
p=0.002\end{array}$ & $\begin{array}{l}c^{2}=12.92 \\
p=0.002\end{array}$ & - \\
\hline \multirow{2}{*}{$\begin{array}{c}\text { Fas } \\
(-1377 \mathrm{G} / \mathrm{A}), \mathrm{n}(\%)\end{array}$} & GA & $6(24.0)$ & $5(12.82)$ & $9(30.0)$ & $4(15.38)$ & \multirow{2}{*}{$\begin{array}{l}c^{2}=3.55 \\
p>0.05\end{array}$} \\
\hline & GG & $19(76.0)$ & $34(87.18)$ & $21(70.0)$ & $22(84.62)$ & \\
\hline \multicolumn{2}{|l|}{$\begin{array}{l}c^{2} \\
p\end{array}$} & $\begin{array}{l}c^{2}=72,20 \\
p<0,001\end{array}$ & $\begin{array}{l}c^{2}=43.13 \\
p<0.001\end{array}$ & $\begin{array}{l}c^{2}=9.60 \\
p=0.002\end{array}$ & $\begin{array}{l}c^{2}=24.92 \\
p<0.001\end{array}$ & - \\
\hline
\end{tabular}

TABLE 7. Association of polymorphic variants of the BCL-2 (rs17759659) gene with the degree of the thyroid gland hyperplasia and its function

\begin{tabular}{|c|c|c|c|c|c|c|}
\hline \multirow{2}{*}{\multicolumn{3}{|c|}{ The genes under study, $n$ (\%) }} & \multicolumn{3}{|c|}{ The thyroid gland function, $\mathrm{n}(\%)$} & \multirow{3}{*}{$\begin{array}{c}\mathbf{c}^{2} \\
\mathbf{p} \\
p=0.029\end{array}$} \\
\hline & & & \multirow{2}{*}{$\begin{array}{c}\begin{array}{c}\text { Euthyroid goiter, } \\
\mathbf{n = 3 1}\end{array} \\
4(12.90)\end{array}$} & \multirow{2}{*}{$\begin{array}{c}\begin{array}{c}\text { Subclinical hypothy- } \\
\text { roidism, } n=71\end{array} \\
1(1.41)\end{array}$} & \multirow{2}{*}{$\begin{array}{c}\text { Clinical hypothyroid- } \\
\text { ism, } \mathrm{n}=\mathbf{2 3}\end{array}$} & \\
\hline \multirow{9}{*}{$\begin{array}{c}B C L-2(\mathrm{~A} / \mathrm{G}) \\
\mathrm{n}(\%)\end{array}$} & \multirow{3}{*}{$A A, \mathrm{n}=10$} & I degree, $n=5$ & & & & \\
\hline & & II degree, $n=1$ & 0 & $1(1.41)$ & 0 & - \\
\hline & & III degree, $n=4$ & 0 & $2(2.82)$ & $2(8.70)$ & $p>0.05$ \\
\hline & \multirow{3}{*}{$G A, n=110$} & I degree, $n=52$ & $23(74.19)$ & $24(33.80)$ & $5(21.74)$ & $\begin{array}{l}c^{2}=19.07 \\
p<0.001\end{array}$ \\
\hline & & II degree, $n=38$ & $3(9.68)$ & $33(46.48)$ & $2(8.70)$ & $\begin{array}{l}c^{2}=20.09 \\
p<0.001\end{array}$ \\
\hline & & III degree, $n=20$ & 0 & $8(11.27)$ & $12(52.17)$ & $p<0.001$ \\
\hline & \multirow{3}{*}{$\mathrm{GG}, \mathrm{n}=5$} & I degree, $n=2$ & $1(3.22)$ & $1(1.41)$ & 0 & $p>0.05$ \\
\hline & & Il degree, $n=1$ & 0 & $1(1.41)$ & 0 & - \\
\hline & & III degree, $n=2$ & 0 & 0 & $2(8.70)$ & - \\
\hline
\end{tabular}


The patients with clinical hypothyroidism were more often recorded with the third degree hyperplasia of the thyroid gland, including those with predominant heterozygous AG-genotype of $\mathrm{BCL}-2$ and CTLA-4 genes by $40.90 \%(p<0.001)$ and $26.21 \%$ $(p=0.002)$, respectively; GG-genotype of Fas gene - by $55.36 \%\left(c^{2}=29.70 ; p<0.001\right)$ (tab. 7-8).

TABLE 8. Association of polymorphic variants of the CTLA-4 (rs231775) and APO-1 / Fas (rs2234767) genes with the degree of the thyroid gland hyperplasia and its function

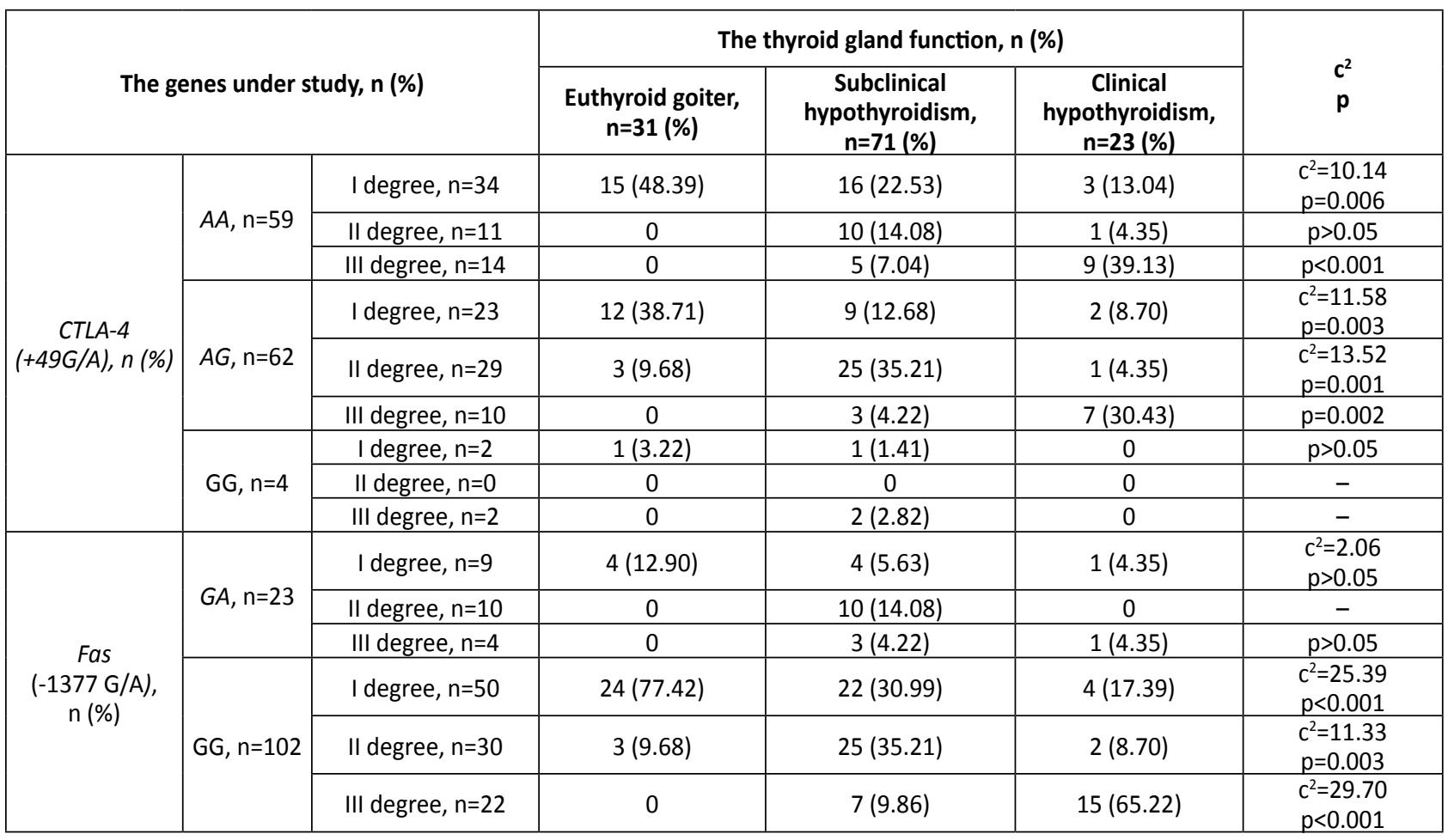

\section{CONCLUSIONS}

TA and NGAIT are more common in the carriers of the minor G-allele (GA- and GG-genotypes) of the $B C L-2$ gene and in homozygous ones having the main $G$ allele (GG-genotype) of the Fas gene by 11.5 and 4.34 times $(p<0.001)$, with no significant interdependence between the genotypes of the CTLA4 gene. We did not find any difference between the relative incidences of the genotypes of the analyzed genes in the patients with NGAIT and those with TA or depending on the TG function (euthyroid goiter, subclinical and clinical hypothyroidism). Hyperplasia of the TG in the patients in general as well as in those with NGAIT is associated with the wild A-alleles of the CTLA-4 gene ( $A A$ - and $A G$-genotypes): the I and III degree hyperplasia occurred reliably more frequently in carriers of the AA genotype by $30.13 \%$ and $26.35 \%$ $\left(c^{2}=9.26 ; p=0.01\right)$, and II degree of the TG enlargement in the patients with AG genotype by $33.52 \%$ and $34.04 \%\left(c^{2}=12.34 ; p=0.002\right)$, respectively. In patients with NGAIT or TA, the TG function is asso- ciated with its hyperplasia and with polymorphic sites of the genes under study. In patients with euthyroid goiter the I degree thyroid hyperplasia is more common: by $11.49-52.45 \%$ ( $p \leq 0.029-0.001$ ) in the carriers of the wild $A$-allele of the $B C L-2$ gene and by $11.49-52.45 \%$ ( $p \leq 0.029-0.001)$ in those with $A$-allele of the CTLA-4 gene and by $46.43 \%$ and $60.03 \%(p<0.001)$ with favorable GG-genotype of the Fas gene. In patients with clinical hypothyroidism, the III degree thyroid hyperplasia is more common: the carriers of the heterozygous AG-genotypes of the $B C L-2$ and $C T L A-4$ genes prevail by $40.90 \%(p<0.001)$ and $26.21 \%(p=0.002)$ respectively, and GG-genotype of the Fas gene by $55.36 \%(p<0.001)$.

\section{Acknowledgement}

This research is part of a first author's doctoral dissertation submitted to I.Y. Horbachevsky State Medical University, Ternopol, Ukraine, under the direction of Professors L.P. Sydorchuk and V.O. Shidlovskyi. 


\section{REFERENCES}

1. Davies TF, Latif R, Yin X. New genetic insights from autoimmune thyroid disease. J Thyroid Res. 2012; 2012: 623852.

2. Simmonds MJ. GWAS in autoimmune thyroid disease: redefining our understanding of pathogenesis. Nat Rev Endocrinol. 2013 May; 9(5): 277-287.

3. Jankovic B, Le KT, Hershman JM. Clinical Review: Hashimoto's thyroiditis and papillary thyroid carcinoma: is there a correlation? J Clin Endocrinol Metab. 2013 Feb; 98(2): 474-482.

4. Sheremet MI, Sydorchuk LP, Shidlovskyi VO, Bedenyuk AD. Research of prognostic markers of proliferation and apoptosis in patients with nodular goiters combined with autoimmune thyroiditis. Archives of the Balkan Medical Union. 2016; 51(4): 488-491.

5. Tsyganenko OS, Voroschuk RS. Immunomorfological reaction in the thyroid tissue in patients with autoimmune thyroiditis in combination with nodular goiter. Arta Medica. Nicholas Anestiadi, Tenth Congress of the Association of Surgeons of Moldova, Chisinau. 2007; 4(25): 51-52.

6. Sheremet MI, Sydorchuk LP, Shidlovskyi VO, Bedenyuk AD et al. New prognostic markers of nodular forms of goiter combined with autoimmune thyroiditis. Journal of Education, Health and Sport 2017; 7(3): 475-482.

7. Sydorchuk LP, Sydorchuk AR, Sheremet $\mathrm{Ml}$, Sydorchuk RI et al. Cytokines cascade changes in patients with rheumatoid arthritis depending on endothelial no-synthase (T-786C) genes polymorphism. Archives of the Balkan Medical Union 2017; 52 (1): 32-38.

8. Kochetova OV. DIO2, TPO, CYP1A1 AND CYP1A2 gene polymorphism in women with thyroid disease. In Kochetova OV, Gaynullina MK, Viktorova TV. Gig Sanit, 2014, 3: 52-56.
9. Gözü HI, Özçelik S, Aloğlu M et al. Is the TSHR D727E polymorphism a genetic predisposition for multinodular goiter in the Turkish population? Genet Mol Res 2016, 15 (3):385-390.

10. Brix TH, Hegedus L. Twin studies as a model for exploring the aetiology of autoimmune thyroid disease. Clin Endocrinol (Oxf). 2012; 76(4): 457-464.

11. Tomer Y, Davies TF. Searching for the autoimmune thyroid disease susceptibility genes: from gene mapping to gene function. Endocr Rev 2003; 24(5): 694-717.

12. Nikitin YP, Rymar OD, Maksimov VN, et al. Association of the T-cell regulatory gene CTLA-4 with susceptibility to autoimmune thyroid disease in population of Novosibirsk. Clinical and experimental thyroidology. 2008; 4(4): 41-45. (in Russian).

13. Zhang M, Ni J, Xu WD et al. Association of CTLA-4 variants with susceptibility to inflammatory bowel disease: A metaanalysis. Hum Immunol. 2014, 75 (3): 227-233.

14. Qiu H, Tang W, Yin P, Cheng F, Wang L. Cytotoxic T-lymphocyte associated antigen 4 polymorphism and Hashimoto's thyroiditis susceptibility: A meta-analysis. Endocrine 2014, 45 (2): 198-205.

15. Biktagirova EM, Kravtsova OA, Sattarova LI, Vagapova GR. Influence of polymorphisms of CTLA-4 and PTPN-22 genes on developmental risk autoimmune thyroiditis among the population of the Republic of Tatarstan. Medical immunology. 2010; 12(1-2): 103-114 [in Russian].

16. Pastuszak-Lewandoska D, Sewerynek E, Domańska D et al. CTLA-4 gene polymorphisms and their influence on predisposition to autoimmune thyroid diseases (Graves' disease and Hashimoto's thyroiditis). Arch Med Sci 2012, 8(3):415421.
17. Lee YH, Choi SJ, Ji JD, Song GG. CTLA-4 and TNF- $\alpha$ promoter-308 A/G polymorphisms and ANCA-associated vasculitis susceptibility: A meta-analysis. Mol Biol Rep. 2012, 39 (1):319-226.

18. Ni J, Qiu LJ, Zhang M et al. CTLA-4 CT60 (rs3087243) polymorphism and autoimmune thyroid diseases susceptibility: a comprehensive meta-analysis. Endocr Res. 2014; 39 (4): 180-188.

19. Sydorchuk LP, Iftoda OM, Kushnir OV. Genes polymorphism of connexin 26 (GJB2) and interleukin 4 (C-590T) in children of Bukovina with hearing loss. Likarska Sprava 2017, 5-6(1139):25-30. [Article in Ukrainian. Abstract in English available].

20. Sydorchuk LP, Amosova KM. Influence of pharmacogenetically determined treatment on parameters of peripheral hemodynamics in patients with arterial hypertension. The New Armenian Medical J 2011; 5(2): 35-43.

21. Leithäuser $F$, Dhein J, Mechtersheimer $G$ et al. Constitutive and induced expression of APO-1, a new member of the nerve growth factor/tumor necrosis factor receptor superfamily, in normal and neoplastic cells. Lab Invest 1993, 69: 415-429.

22. Man-Man Lu, Qian-Ling Ye, Chen-Chen Feng et al. Association of FAS gene polymorphisms with systemic lupus erythematosus: A case-control study and meta-analysis. Experimental and Therapeutic Medicine 2012; 4: 497-502.

23. Tomer Y. Genetic susceptibility to autoimmune thyroid disease: Past, present, and future. Thyroid. 2010; 20(7): 715-725.

24. Dong YH, Fu DG. Autoimmune thyroid disease: Mechanism, genetics and current knowledge. Eur Rev Med Pharmacol Sci. 2014; 18(23): 3611-3618. 